\title{
Optimizing the operation of a direct-flow filtration device
}

\author{
James G. Herterich • Qian Xu • \\ Robert W. Field · Dominic Vella • \\ Ian M. Griffiths
}

Received: 24 May 2016 / Accepted: 1 October 2016 / Published online: 8 December 2016

(C) The Author(s) 2016. This article is published with open access at Springerlink.com

\begin{abstract}
Direct-flow filtration is a common technique for filtering impurities from a fluid using a porous-walled channel or a pipe, one end of which is closed off with a cap. Pure fluid flows out of the porous walls, while impurities are left in the channel. Such systems are composed of a series of individual porous channels or pipes stacked in close proximity. We develop a mathematical model for the flow in a 2D filtration channel and a 3D pipe, with a capped end, to describe the behaviour within a direct-flow device. We study the axial dependence of the transmembrane pressure (TMP) across the membrane walls on the imposed flux, wall permeability and the proximity of the neighbouring fibres. The mathematical models derived are used to predict the operating regimes of the device that maximize the spatial uniformity in the TMP and thus optimize the use of the entire membrane area. We show how a large portion of the available membrane area is not used when the fibres are packed too closely together, with the majority of the filtration behaviour being localized near to the impermeable capped end; this leads to inefficient filtration. We quantify the device performance by examining the uniformity of the filtration across the length of the device and the output of the filtered fluid for a given operating pressure.
\end{abstract}

Keywords Blocked end · Direct-flow filtration · Hollow fibre $\cdot$ Transmembrane pressure

\section{Introduction}

Membrane filtration is one of the most common methods for purifying fluids [1,2]. Here, a fluid containing contaminants is pushed through a porous membrane; the fluid penetrates the membrane, while the contaminants are retained. Membrane filtration typically takes place either in dead-end or crossflow modes (Fig. 1a, b). In classical dead-end

James G. Herterich and Qian Xu contributed equally to this study

J. G. Herterich · D. Vella · I. M. Griffiths $(\bowtie)$

Mathematical Institute, University of Oxford, Woodstock Road, Oxford OX2 6GG, UK

e-mail: ian.griffiths@maths.ox.ac.uk

Q. Xu · R. W. Field

Department of Engineering Science, University of Oxford, Parks Road, Oxford OX1 3PJ, UK

J. G. Herterich

School of Mathematics and Statistics, University College Dublin, Belfield, Dublin 4, Ireland 


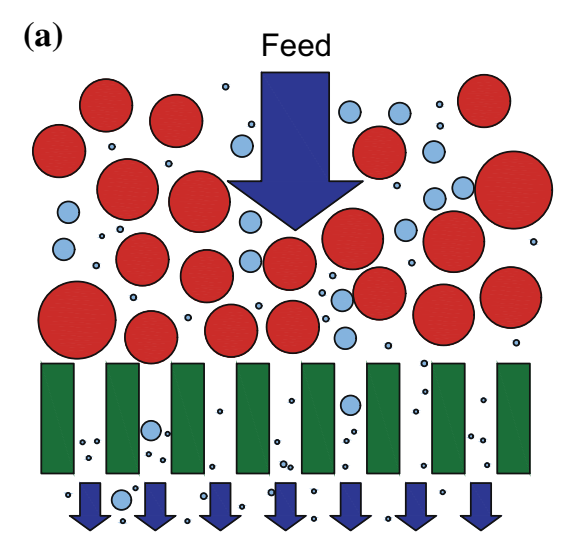

Permeate (b)

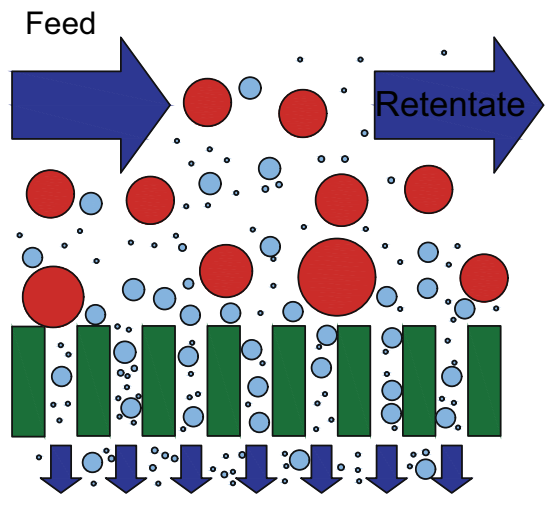

Permeate

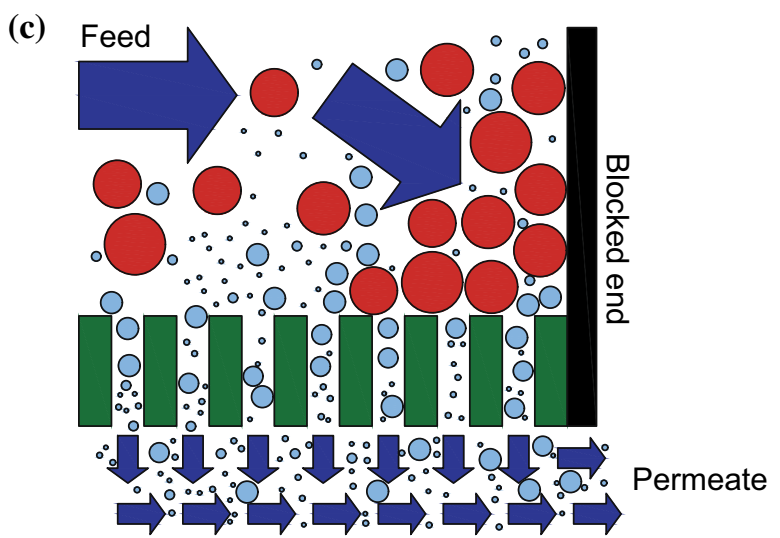

Fig. 1 Schematics of a dead-end, b crossflow and $\mathbf{c}$ direct-flow filtrations. Typically, the respective permeate rates are high, low and moderate, while the rates of fouling are the opposite, i.e. high, low and moderate

filtration mode, both the flow and the filtration direction are normal to the membrane surface; in crossflow mode, the feed flow is parallel to the membrane surface, while permeation occurs naturally through the walls, orthogonal to the flow [3]. In both cases, contaminants build up on the surface and reduce the permeability of the membrane to the fluid, as filtering progresses. This effect, termed concentration polarization (or fouling when the contaminants physically block the membrane pores) poses one of the most significant challenges limiting membrane filtration [4-6]. Concentration polarization is greatly reduced in crossflow filtration, due to the flow occurring tangential to the membrane, which sweeps the contaminants downstream and limits build-up of contaminants at the surface. The fluid that is not transported across the membrane is re-circulated. This advantage requires higher input fluxes to generate the same quantities of purified fluid as in dead-end filtration.

Direct-flow filtration offers an efficient combination of the benefits of both dead-end and crossflow filtrations. This is achieved by capping the end of a crossflow device so that all the fluid is forced to pass through the walls (Fig. 1c). Direct-flow filtration is more energy efficient than crossflow filtration and so forms the dominant method for producing potable water from ground or surface water [7]. In industrial filtration, a direct-flow module commonly consists of hundreds of hollow fibres arranged in a 3D cylindrical fashion (Fig. 2) [8]. Since the permeate is shared by each fibre, the flow through each channel is coupled to that in the others.

Unlike in crossflow filtration, fouling is not controlled via recirculation but can be minimized by incorporating very short automatic backwashes at regular frequencies [3-5], in which the imposed pressure gradient (and hence the flow) is reversed to dislodge the contaminants that have built up at the surface [9-11]. The lack of recirculation 


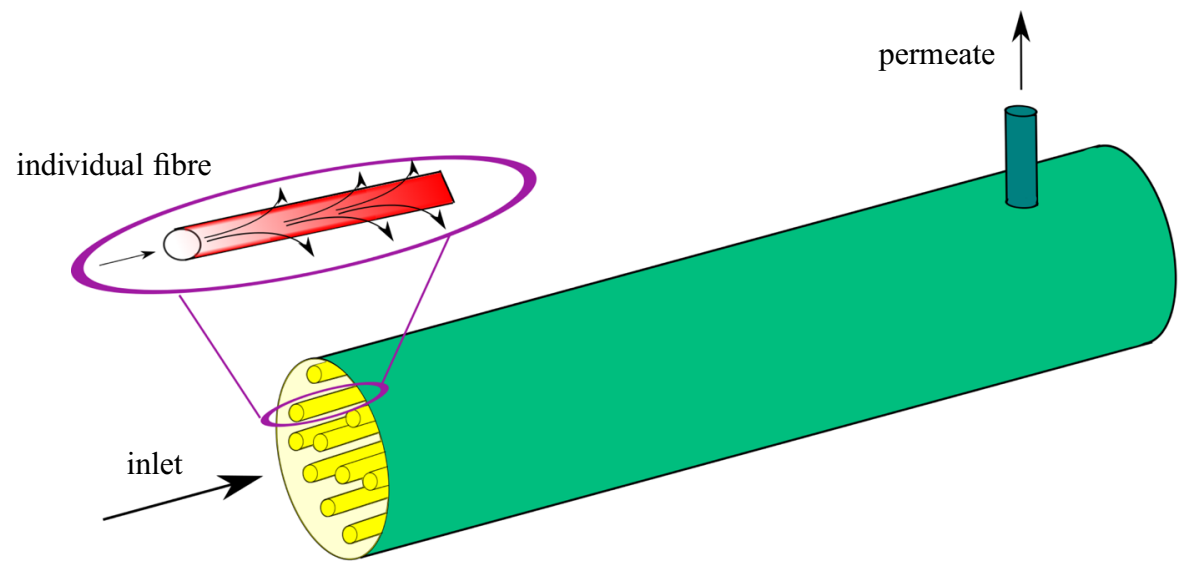

Fig. 2 Schematic of a hollow-fibre filtration device as used in direct-flow filtration

Table 1 Summary of flow situations in three filtration modes

\begin{tabular}{llll}
\hline & Dead-end & Crossflow & Direct-flow \\
\hline Strength of feed flow & Feed flow = Permeate flow & Feed flow $\gg$ Permeate flow & Feed flow = Permeate flow \\
Tangential flow & None & Yes, strong & Yes, weak \\
$\begin{array}{l}\text { Orientation of permeate with } \\
\text { regard to feed }\end{array}$ & Same direction & Orthogonal & Orthogonal \\
\hline
\end{tabular}

in direct-flow filtration results in lower flow rates (and thus a lower axial pressure gradient) to achieve permeate fluxes comparable to those of crossflow filtration, and so many finer feed channels may be used [12]. A comparison of the three filtration modes is given in Table 1.

In all filtration scenarios, the main quantity of interest is the volumetric flux across the porous wall, which depends on the local transmembrane pressure (TMP): the more spatially uniform the TMP, the more effectively the membrane area is used for filtration. The TMP is a complex function of operating flux, membrane wall permeability and, crucially, the packing density of the fibres within a device [13]. Hurwitz [14] modelled the direct-flow process by considering the flow inside a single porous tube with a capped end. The flow was solved for in the asymptotic limits of low permeability and low Reynolds number. Sanaei et al. [15] derive a theoretical model for the flow behaviour in a pleated filter, composed of a series of permeable pleated 'fingers'. The impact of pore constriction on filter blocking as a result of this configuration was also explored. In both Hurwitz [14] and Sanaei et al. [15], the coupling that results from the flow on the permeate side was not considered. Griffiths et al. [16] address the question of how to choose the spatial dependence of the wall permeability to allow for the uniform delivery of solute (nutrient) across the permeable wall in a crossflow device.

Although many authors have studied the impact of particle accumulation on the overall filtration output, little emphasis has been placed on explaining the influence of pressure profiles on the direct-flow filtration process. This paper provides a mathematical approach demonstrating how the pressure profile changes in response to fibre spacing and operating conditions for these devices in the absence of fouling. Since fouling is flux driven, a spatially uniform pressure drop helps to mitigate the influence of fouling and extend filter lifetime [11,17]. Indeed, Alfa-Laval introduced in the early 1990s the Bactocatch process with feed and permeate flowing co-currently in order to achieve an even transmembrane pressure profile $[18,19]$. However, a similar approach would be too energy intensive in the water industry where direct flow finds its main application; nevertheless, the desire to have as even a transmembrane pressure as possible remains. 
In this paper, we develop a mathematical model for the fluid flow in a direct-flow filter. The feed and permeate flow in a single fibre of the direct-flow device are modelled analytically, by exploiting the slenderness of the fibres [20], with a transmembrane pressure difference across the porous fibre walls. Initially, a $2 \mathrm{D}$ analogue is analysed as a convenient way of introducing our analysis. We then turn our attention to a 3D cylindrical configuration for the direct-flow device to model the appropriate configuration for water-treatment systems. We study the uniformity with which the fluid passes across the membrane area and how this is affected by the proximity of the neighbouring fibres. We use the resulting model to predict the optimal operation configuration that offers the appropriate balance between maximizing the membrane area used while minimizing the space occupied by the entire device.

\section{2D Channel model development}

\subsection{Setup}

We begin by considering a 2D analogue of the direct-flow device in which the flow enters a single 2D channel, of length $L$ and width $H$, with permeable walls. The wall conductivity, $\hat{k}$, is constant, and the end of the channel is capped (impermeable), as shown in Fig. 3. We use a Cartesian coordinate system $(\hat{x}, \hat{z})$ to represent the system, where $\hat{x}$ denotes the direction perpendicular to the permeable channel walls and $\hat{z}$ denotes the distance along the channel. Fluid enters the channel (Region 1) at a fixed flux $2 \hat{Q}$, which passes through the membrane side walls, and into the permeate region (Region 2). For an isolated channel, we would consider Region 1 inside the channel and a quiescent bath outside. However, since a typical direct-flow device is composed of a series of such channels packed together, the flow in Region 2 must also be considered. Since the end of the channel is blocked, the entire fluid must eventually flow through the permeable walls from Region 1 into Region 2 (net flux $\hat{Q}$ through each side of the channel by symmetry).

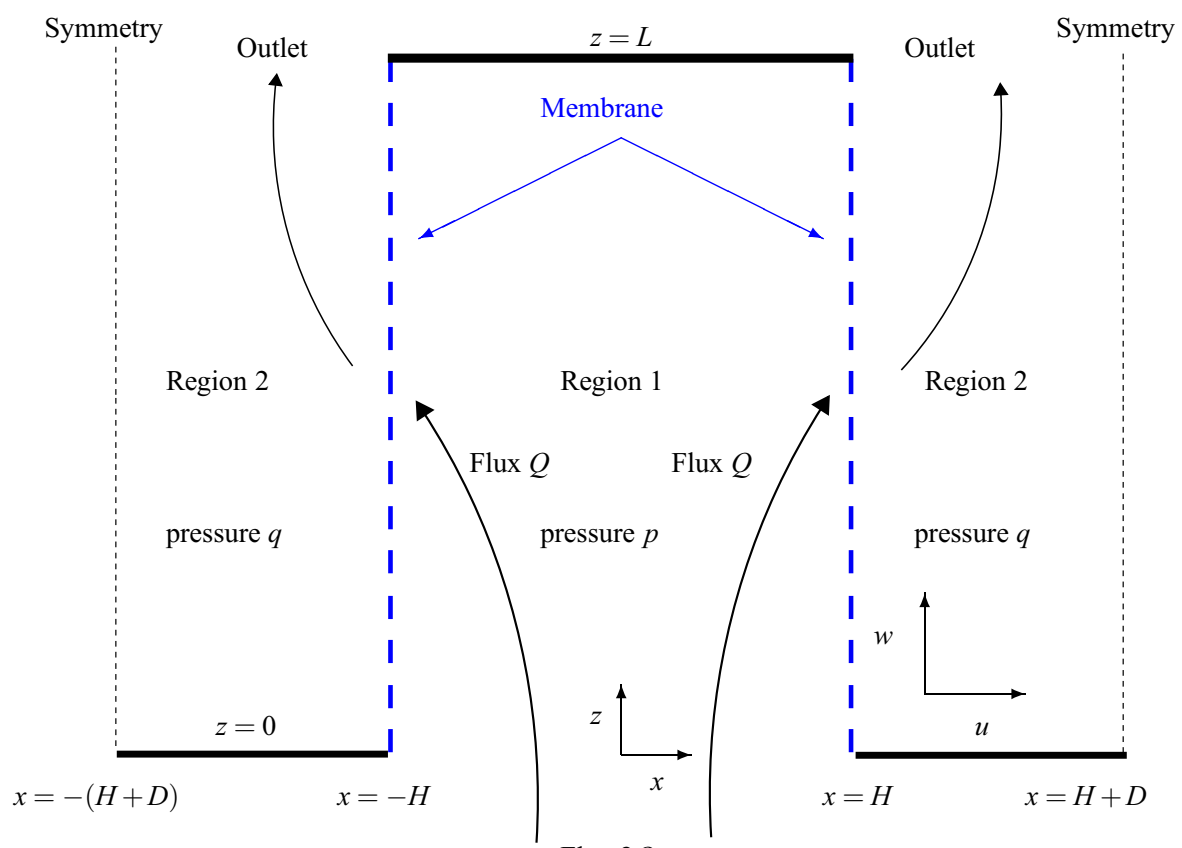

Flux $2 Q$

Fig. 3 Schematic of the flow within a single direct-flow 2D channel. Solid lines indicate impermeable walls, blue dashed lines indicate permeable walls, and the dotted line indicates symmetry in the flow. Flow enters from the bottom and passes through the permeable walls. Beyond the capped end, the fluid continues to an outlet following the black arrows. The inside of the membrane-walled channel is referred to as Region 1, while that outside is Region 2. (Color figure online) 
We consider an array of such channels with a centre-centre separation $2(H+D)$ and so impose a symmetry condition at $\hat{x}= \pm(H+D)$. The fluid velocity field in the $(\hat{x}, \hat{z})$ directions is $\hat{\mathbf{u}}=(\hat{u}(\hat{x}, \hat{z}), \hat{w}(\hat{x}, \hat{z}))$, while the (spatially variable) pressure inside the channel is $\hat{p}$ with that outside the channel denoted by $\hat{q}$. The quantity of most interest is the transmembrane pressure difference (TMP), $\hat{p}-\hat{q}$ at $\hat{x}= \pm \hat{H}$; The greater the spatial uniformity in the TMP, the more uniformly the membrane area is used for filtration.

Typically, for hollow-fibre membrane module configurations, $L=1.5 \mathrm{~m}$ and $2 H=0.7-1 \mathrm{~mm}$, so that the aspect ratio, $\delta=H / L=O\left(10^{-3}\right)$. Since the membrane is thin (e.g. typical thicknesses $h \sim 10 \mu \mathrm{m}$ [21] and so $h / H \sim 10^{-3}$ ), we can ignore the behaviour within the membrane when solving for the flow. Furthermore, the effect of the membrane thickness on the pressure drop across the membrane is captured in the effective permeability parameter, $\hat{k}$, which we vary in our analysis.

The Reynolds number of this channel is defined as $\operatorname{Re}=(2 H \rho W) / \mu$, where $\rho$ is the density of the fluid (water considered in this paper), $W$ is the typical axial velocity $(\mathrm{cm} / \mathrm{s})$, and $\mu$ is the dynamic viscosity. In this case, $R e \approx 20$, however, because $\delta=H / L \approx 5 \times 10^{-4}$ the reduced Reynolds number $\delta R e=O\left(10^{-2}\right) \ll 1[12]$.

In principle, the particles contained within the fluid will influence the flow field. However, the influence on the flow field is small for dilute concentrations [20], or higher concentrations of larger particles (such as in microfiltration) where osmotic pressures are lower [1]. The particles will also affect the flow as they accumulate on and within the membrane, causing blocking and flow redistribution. There are typically two to three feed/standard hydraulic backwash cycles per hour, one to three chemically enhanced backwashes per day and a major clean only once per month. Since the typical time taken for fluid to pass through the device is on the order of a minute, the net accumulation and indeed accumulation during any one cycle is small. As a result, in this paper, we focus our attention on the fluid dynamics in a steady-state configuration, neglecting the start-up process and the longer-term effect that particles may have on this.

\subsection{Governing equations}

Since the reduced Reynolds number is small and the channel is thin, the fluid flow obeys the lubrication equations [20]. The symmetry of the $2 \mathrm{D}$ channel means that we need only to solve the system for $(\hat{x}, \hat{z}) \in([0, H+D],[0, L])$.

We non-dimensionalize lengths with the channel length, $L$, and exploit the small aspect ratio, $\delta=H / L \ll 1$. Similarly, the velocities are scaled with the typical axial velocity, $W$ :

$$
\begin{aligned}
& \hat{z}=L z, \quad \hat{x}=\delta L x, \quad \hat{w}=W w, \quad \hat{u}=\delta W u, \quad \hat{p}=\frac{\mu W}{\delta^{2} L} p, \quad \hat{q}=\frac{\mu W}{\delta^{2} L} q, \\
& \hat{Q}=\delta W L Q, \quad \hat{k}=\frac{\delta^{3} L}{\mu} k .
\end{aligned}
$$

In this model, we allow for variations in the dimensionless flux, $Q$, the effective membrane permeability, $k$, and the parameter $\ell=D / H$, which characterizes the dimensionless inter-fibre spacing. Note that we have chosen not to scale the velocity with $\hat{Q} / L$ so that the effect of flux remains explicitly in the resulting system.

In Region $1(0<x<1$ and $0<z<1)$, we have the lubrication equations

$$
\begin{aligned}
& \frac{\partial u}{\partial x}+\frac{\partial w}{\partial z}=0, \\
& \frac{\partial^{2} w}{\partial x^{2}}=\frac{\mathrm{d} p}{\mathrm{~d} z} .
\end{aligned}
$$

These equations are subject to typical boundary conditions that reflect the symmetry of the flow, the flux of fluid and no flow through the impermeable end. We assume a filtration velocity that is proportional to the transmembrane pressure difference and permeability of the membrane (i.e. Darcy flow) [22]. In general, at a permeable wall there is a 
tangential slip velocity, whose magnitude is determined by a Neumann boundary condition such as that given in [23]. However, it has been found that this slip is not significant for a wide range of membranes [24,25] and so here, for simplicity, we shall assume a no-slip boundary condition. Mathematically the boundary conditions may be expressed as

\begin{tabular}{|c|c|c|}
\hline Symmetry: & $\frac{\partial w}{\partial x}=u=0$ & on $x=0$, \\
\hline No axial wall slip: & $w=0$ & on $x=1$, \\
\hline Permeate flow: & $u=k(p-q)$, & on $x=1$, \\
\hline Fluid flux: & $\int_{0}^{1} w \mathrm{~d} x=Q$ & at $z=0$, \\
\hline No fluid outflow: & $w=0$ & at $z=1$. \\
\hline
\end{tabular}

As the end is capped all injected fluid must pass through the membrane, and so

$\int_{0}^{1} u \mathrm{~d} z=Q$ at $x=1$

In this model, there is a transverse flow, $u$, at the capped end, $z=1$. In reality, there is a no-slip condition here $(u=0)$. As a result of exploiting the slenderness of the system geometry, the order of the system is reduced and we are not able to impose this additional constraint. However, this no-slip condition influences the flow only in a boundary layer of size $\delta$ near the capped end (where we would have to solve the full Stokes equations, as considered in [14]) and so we are able to neglect this effect in our model.

In Region $2(1<x<1+\ell$ and $0<\mathrm{z}<1)$, the fluid also obeys the lubrication equations

$$
\begin{aligned}
& \frac{\partial u}{\partial x}+\frac{\partial w}{\partial z}=0, \\
& \frac{\partial^{2} w}{\partial x^{2}}=\frac{d q}{d z}
\end{aligned}
$$

The boundary conditions reflect the symmetry of the flow, no-slip at the porous wall, continuity of velocity across

\begin{tabular}{|c|c|c|}
\hline Symmetry: & $\frac{\partial w}{\partial x}=u=0$ & on $x=1+\ell$ \\
\hline No axial wall slip: & $w=0$ & on $x=1$ \\
\hline Permeate flow: & $u=k(p-q)$ & on $x=1$ \\
\hline No fluid flow: & $w=0$ & at $z=0$, \\
\hline Fixed outlet pressure: & $q=0$ & at $z=1$. \\
\hline
\end{tabular}
the membrane, the impermeable bottom, and a fixed outlet pressure, i.e.

Note that the permeate-flow boundary conditions (3c) and (6c) are the same, enforcing continuity of flux across the membrane.

\subsection{Flow in a direct-flow device}

The system of equations (2), (3), (5), and (6) can be solved analytically. Using both sets of symmetry and no axial wallslip conditions, (3a, b) and (6a, b), we can express the fluid velocities in terms of the pressures. In Region 1, we have 


$$
\begin{aligned}
& w=\frac{1}{2} \frac{d p}{d z}\left(x^{2}-1\right), \\
& u=\frac{1}{6} \frac{d^{2} p}{d z^{2}}\left(3-x^{2}\right) x,
\end{aligned}
$$

while in Region 2,

$$
\begin{aligned}
& w=\frac{d q}{d z}\left[\frac{1}{2} x^{2}-(1+\ell) x+\ell+\frac{1}{2}\right] \\
& u=\frac{d^{2} q}{d z^{2}}\left[-\frac{1}{6} x^{3}+\frac{1}{2}(1+\ell) x^{2}-\left(\ell+\frac{1}{2}\right) x-\frac{1}{3}(1+\ell)^{3}+\left(\ell+\frac{1}{2}\right)(\ell+1)\right] .
\end{aligned}
$$

In each region, we find a Poiseuille profile for the axial velocity, and a transverse velocity that is proportional to the second pressure derivative and is cubic in the coordinate $x$ [26]. However, we shall see that the pressure itself is affected by the capped end of the channel.

Substituting the transverse velocity expressions, (7b) and (8b), into the boundary conditions for the permeate flux through the membrane, (3c) and (6c), gives a set of second-order ordinary differential equations (ODEs) for the pressures, $p$ and $q$. Using the axial velocities in (7a) and (8a) we can rewrite the boundary conditions (3) and (6) to obtain conditions for $p$ and $q$. The flux condition (3d) becomes

$$
\left.\frac{\mathrm{d} p}{\mathrm{~d} z}\right|_{z=0}=-3 Q,
$$

while (3e) and (6d) become

$$
\left.\frac{\mathrm{d} p}{\mathrm{~d} z}\right|_{z=1}=0,\left.\quad \frac{\mathrm{d} q}{\mathrm{~d} z}\right|_{z=0}=0,
$$

respectively.

The complete system then reads

$$
\begin{array}{ll}
\frac{1}{3} \frac{\mathrm{d}^{2} p}{\mathrm{~d} z^{2}}=k(p-q), & \left.\frac{\mathrm{d} p}{\mathrm{~d} z}\right|_{z=0}=-3 Q,\left.\quad \frac{\mathrm{d} p}{\mathrm{~d} z}\right|_{z=1}=0, \\
\frac{l^{3}}{3} \frac{\mathrm{d}^{2} q}{\mathrm{~d} z^{2}}=k(q-p), & \left.\frac{\mathrm{d} q}{\mathrm{~d} z}\right|_{z=0}=0, \quad q(z=1)=0 .
\end{array}
$$

The system of ODEs (11) may be solved analytically to give

$$
\begin{aligned}
& p(z)=\frac{1}{1+\ell^{3}}\left[\ell^{3} \mathcal{M}(z)+3 Q(1-z)+\mathcal{M}_{0}\right], \\
& q(z)=\frac{1}{1+\ell^{3}}\left[-\mathcal{M}(z)+3 Q(1-z)+\mathcal{M}_{0}\right],
\end{aligned}
$$

where

$$
\begin{aligned}
\mathcal{M}(z) & =\operatorname{sech}\left(C_{1}\right)\left\{\mathcal{M}_{0} \cosh \left(C_{1} z\right)+\frac{3 Q}{C_{1}} \sinh \left[C_{1}(1-z)\right]\right\}, \\
\mathcal{M}_{0} & =\frac{3 Q}{C_{1}}\left[\frac{1}{\ell^{3}} \operatorname{coth}\left(C_{1}\right)+\operatorname{csch}\left(C_{1}\right)\right],
\end{aligned}
$$


(a)

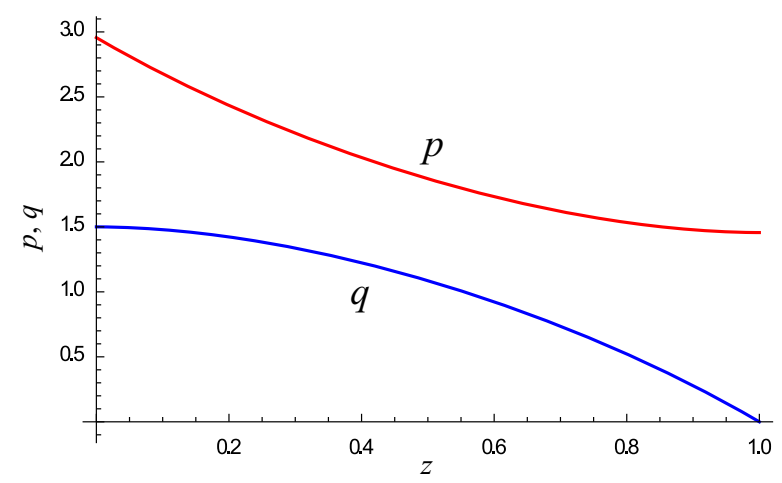

(b)

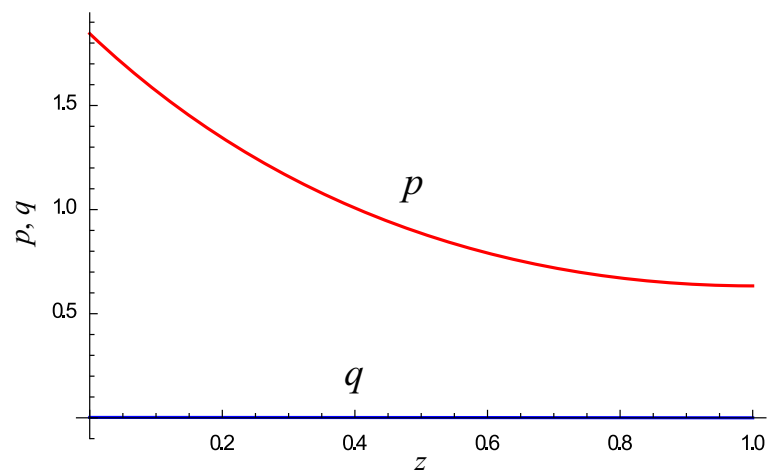

Fig. 4 Variation of fluid pressure inside the channel, $p$ (red), and outside the channel, $q$ (blue) given by (12). The permeability $k=1$ and the flux $Q=1$, with dimensionless module spacing parameters $\mathbf{a} \ell=1$ and $\mathbf{b} \ell=10$. (Color figure online)

$$
C_{1}=\sqrt{3 k\left(1+\frac{1}{\ell^{3}}\right)} .
$$

We now consider the behaviour of the pressures $p$ and $q$ for different module spacing parameters, $\ell$. When $\ell=1$, there is a negative pressure gradient on both sides of the membrane ( $p$ and $q$ are decreasing, as seen in Fig. 4) with the pressure in Region 1 larger than the pressure in Region 2. This means that there is a flow of fluid in the positive $z$-direction on both side of the membrane and a flow through the membrane, as expected. When $\ell=10$, the outside pressure, $q$, is effectively zero everywhere (Fig. 4), equivalent to a quiescent bath (i.e. it is unaffected by the flow from the channel).

As discussed, the quantity of real interest is not $p$ and $q$ separately but rather the TMP, $p-q$, which is simply given by the function $\mathcal{M}(13 \mathrm{a})$ : the more spatially uniform the TMP the more effective the use of the available membrane area. As $Q$ increases, the TMP (13a) increases linearly at each point along the membrane. In general, the TMP has a convex shape with a central minimum, so that more of the fluid is filtered near the entrance and capped end (Fig. 5a). For small values of $Q$, the TMP is small but still relatively large at the ends. When $Q$ is larger, the TMP retains the same qualitative shape, but varies in absolute terms to a larger degree along the membrane. The TMP has a qualitatively similar profile as we vary $k$, although higher pressures are required for smaller permeabilities (Fig. 5b) — this should be expected because in direct-flow with constant flux, all the fluid must be filtered through the membrane. The variation between minimum and maximum TMPs increases with increasing $k$.

Varying the spacing parameter of the channel, $\ell$, affects not only the magnitude of the TMP but also its shape (Fig. 5c). When $\ell>1$, permeate channels are wider than the feed channel $D>H$ and the section of the membrane walls nearest the entrance of the channel has the largest TMP. The TMP then decreases monotonically, as the permeate side behaves like a quiescent bath (Fig. 4b). When $\ell \sim 1$ (i.e. $D \sim H$ ), the TMP assumes a profile with a central minimum. For $\ell<1$, when permeate channels are relatively small compared with the feed channels $(D<H)$, the TMP is the largest at the impermeable capped end. This results in the majority of filtration occurring further down the channel, a situation to be avoided since a large area of the membrane is not being used to its full potential.

The pump-driving pressure at the entrance to the module is given by (12a) evaluated at $z=0$ :

$p(0)=\frac{3 Q\left\{C_{1}+\left[2+\left(\ell^{3}+\frac{1}{\ell^{3}}\right) \cosh C_{1}\right] \operatorname{csch} C_{1}\right\}}{\left(1+\ell^{3}\right) C_{1}}$.

Clearly this depends on the imposed flux, $Q$, the permeability, $k$, and module spacing parameter, $\ell$, through $C_{1}$, (13c). The driving pressure increases linearly with flux, as expected. As both the permeability and separation 
(a)

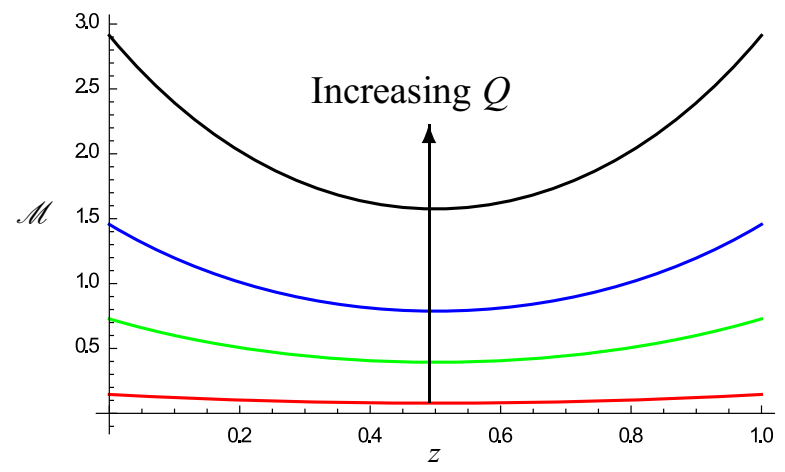

(b)

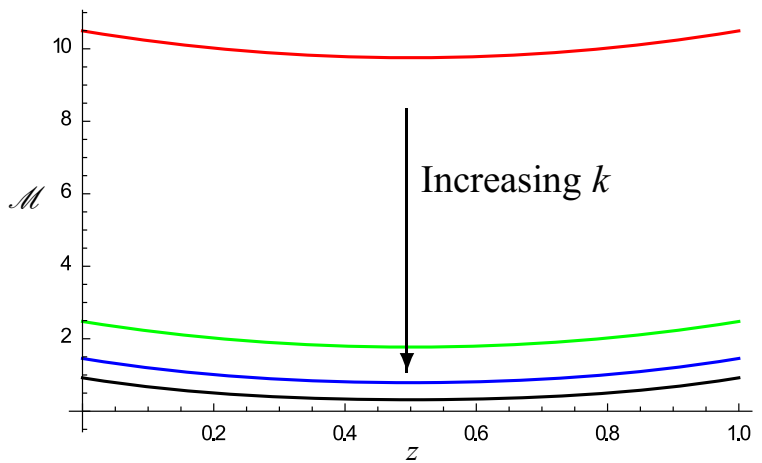

(c)

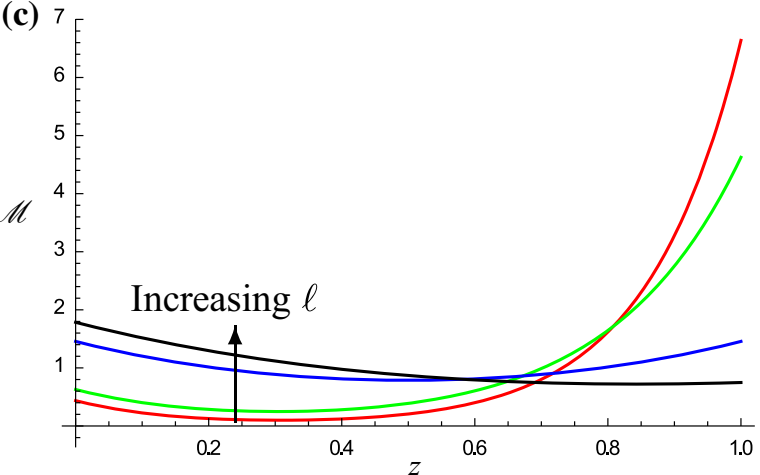

Fig. 5 Profiles of transmembrane pressure difference (TMP), $\mathcal{M}(z)=\left.(p-q)\right|_{x=1}$, in a capped 2D channel. Variation with a flux, $Q$, b permeability, $k$, and c module spacing parameter, $l$. In each figure, the varying quantity takes the values $0.1,0.5,1,2$, plotted in red, green, blue and black respectively, with other quantities taking the value 1. (Color figure online)

distance increase, the driving pressure decreases. This is because a greater pressure is required to force the fluid through the walls when the permeability is small. The behaviour when we vary the module spacing parameter, $\ell$, is explained via the TMP in the system (Fig. 5c). As $\ell$ is decreased, more of the fluid passes through a smaller fraction of the membrane area, and so a higher pressure is required. When $\ell$ is large so that the permeate channels have a small effect on the flow, the pressure difference (13a) takes a form similar to that of a crossflow channel with full fractional recovery (see, e.g. [13]), or a channel with zero net exit flux as in [16].

\section{3D Pipe model development}

In the 3D geometry of relevance industrially, the pipes themselves have a circular cross-section. However, the position of the symmetry boundary is no longer simple and will, in general, depend on the packing of the pipes (square, hexagonal, etc.). For simplicity, we will assume that a symmetry condition may be applied at a particular radial coordinate, so that an axisymmetric coordinate system $(\hat{r}, \hat{z})$ may be used. Here, the radial coordinate $\hat{r}$ replaces the Cartesian coordinate $\hat{x}$, and the $\hat{z}$ coordinate remains unchanged. We denote the radius of Region 1 by $R$ and non-dimensionalize $\hat{r}=R r$ so that $0 \leq r \leq 1$ in Region 1 . The module spacing parameter is now $\ell=D / R$ so that $1 \leq r \leq 1+\ell$ corresponds to Region 2 .

The dimensionless equations analogous to (2), (3), (5), and (6) for a cylindrical geometry may easily be written down, again exploiting the small aspect ratio (which in this case is $R / L$ ). In Region 1, the fluid obeys the lubrication equations, 
$\frac{1}{r} \frac{\partial}{\partial r}(r u)+\frac{\partial w}{\partial z}=0$

$\frac{1}{r} \frac{\partial}{\partial r}\left(r \frac{\partial w}{\partial r}\right)=\frac{\mathrm{d} p}{\mathrm{~d} z}$

for $0<r<1$ and $0<z<1$. The boundary conditions read, by analogy with (3):

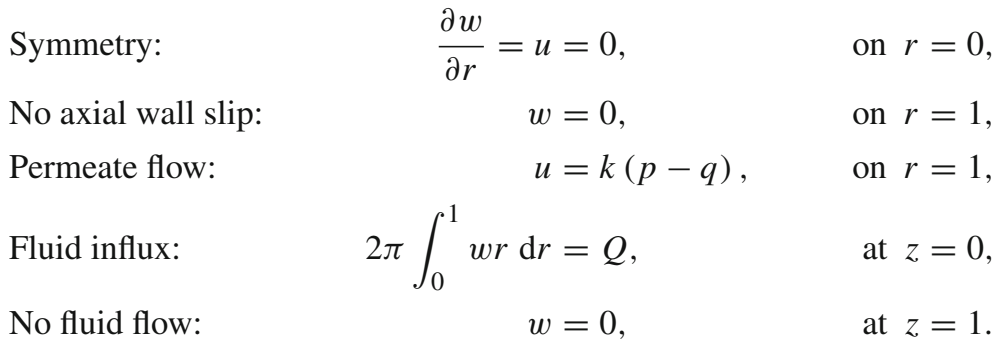

In Region 2, the fluid again obeys the lubrication equations,

$\frac{1}{r} \frac{\partial}{\partial r}(r u)+\frac{\partial w}{\partial z}=0$

$\frac{1}{r} \frac{\partial}{\partial r}\left(r \frac{\partial w}{\partial r}\right)=\frac{\mathrm{d} q}{\mathrm{~d} z}$

for $1<r<1+\ell$ and $0<z<1$. For this region, the boundary conditions read,

\begin{tabular}{|c|c|c|}
\hline Symmetry: & $\frac{\partial w}{\partial r}=u=0$ & on $r=1+\ell$ \\
\hline No axial wall slip: & $w=0$ & on $r=1$, \\
\hline Permeate flow: & $u=k(p-q)$ & on $r=1$, \\
\hline No fluid flow: & $w=0$, & at $z=0$ \\
\hline Fixed outlet pressure: & $q=0$ & at $z=1$. \\
\hline
\end{tabular}

As in the 2D case, the flow in a single element, given by (15)-(18), can be solved analytically. Using both sets of symmetry and no axial wall-slip conditions $(16 a, b)$ and $(18 a, b)$, we can solve for the fluid flow in terms of the pressure gradient. In Region 1, we find

$$
\begin{aligned}
& w=\frac{1}{4} \frac{\mathrm{d} p}{\mathrm{~d} z}\left(r^{2}-1\right), \\
& u=\frac{1}{16} \frac{\mathrm{d}^{2} p}{\mathrm{~d} z^{2}}\left(2 r-r^{3}\right),
\end{aligned}
$$

similar to [14], although the pressure will take a different form since we consider the combined flow in Regions 1 and 2. In Region 2, we find

$$
\begin{aligned}
& w=\frac{1}{4} \frac{\mathrm{d} q}{\mathrm{~d} z}\left[r^{2}-2(1+\ell)^{2} \log r-1\right], \\
& u=\frac{1}{16} \frac{\mathrm{d}^{2} q}{\mathrm{~d} z^{2}}\left[2 r-r^{3}+(1+\ell)^{2}\left(-2 r+4 r \log r-(1+\ell)^{2}\left(-\frac{3}{r}+\frac{4}{r} \log (1+\ell)\right)-\frac{2}{r}\right)\right] .
\end{aligned}
$$


In an analogous fashion to the 2D case, the permeate flux through the membrane, (16c) and (18c) give a set of second-order ordinary differential equations (ODEs) for the pressures $p$ and $q$. These equations have two boundary conditions each, given by

$$
\begin{array}{ll}
\frac{1}{16} \frac{\mathrm{d}^{2} p}{\mathrm{~d} z^{2}}=k(p-q), & \left.\frac{\mathrm{d} p}{\mathrm{~d} z}\right|_{z=0}=-\frac{8 Q}{\pi},\left.\quad \frac{\mathrm{d} p}{\mathrm{~d} z}\right|_{z=1}=0 \\
-\frac{\Lambda}{16} \frac{\mathrm{d}^{2} q}{\mathrm{~d} z^{2}}=k(p-q), & \left.\frac{\mathrm{d} q}{\mathrm{~d} z}\right|_{z=0}=0, \quad q(z=1)=0
\end{array}
$$

where $\Lambda=\Lambda(l)$ is given by

$-\Lambda(\ell)=1-4(1+\ell)^{2}+[3-4 \log (1+\ell)](1+\ell)^{4}<0$.

The ODEs (21) may be solved analytically to yield the pressures, $p$ and $q$, in the axisymmetric pipe:

$$
\begin{aligned}
& p(z)=\frac{1}{1+\Lambda}\left[\Lambda \mathcal{N}(z)+\frac{8 Q}{\pi}(1-z)+\mathcal{N}_{0}\right], \\
& q(z)=\frac{1}{1+\Lambda}\left[-\mathcal{N}(z)+\frac{8 Q}{\pi}(1-z)+\mathcal{N}_{0}\right],
\end{aligned}
$$

where

$$
\begin{aligned}
& \mathcal{N}(z)=\operatorname{sech}\left(C_{2}\right)\left[\mathcal{N}_{0} \cosh \left(C_{2} z\right)+\frac{8 Q}{\pi C_{2}} \sinh \left(C_{2}(1-z)\right)\right], \\
& \mathcal{N}_{0}=\frac{C_{2} Q}{2 \pi k(\Lambda+1)} \operatorname{coth}\left(C_{2}\right)+\frac{8 Q}{\pi C_{2}} \operatorname{csch}\left(C_{2}\right), \\
& C_{2}=\sqrt{16 k\left(1+\frac{1}{\Lambda}\right)} .
\end{aligned}
$$

The TMP in the pipe (Fig. 6), given by the function $\mathcal{N}(z)=\left.(p-q)\right|_{r=1},(24 a)$, behaves similarly to that of the 2D channel, cf., Fig. 5. In this case, however, the TMP at the entrance is typically larger than that at the capped end, except for very small module spacing parameters, $\ell$ (Fig. 6c). In order to achieve approximately constant TMP, as preferred for the uniform use of membrane area, the permeability and flux must be low and the spacing parameter similar to the width of tube $(D \sim H)$.

\section{Discussion of results and optimization}

We have so far considered the behaviour of the pressure profiles in both 2D and 3D (axisymmetric), both inside and outside, for a range of (a) module spacing parameter, $\ell$; (b) fluxes, $Q$; and (c) permeabilities, $k$. In this section, we explore how we may use the mathematical model that we have presented to understand how a direct-flow device can be arranged to operate optimally and, in particular, making good use of the available membrane area.

As $Q$ increases, the TMP increases linearly at each point along the membrane, resulting in a convex profile with a minimum in 2D (Fig. 5) and a descending curve in 3D (Fig. 6). When $Q$ is large and $\ell$ is small, the TMP retains the qualitative shape in both $2 \mathrm{D}$ and $3 \mathrm{D}$, but varies to a larger degree absolutely (i.e. a larger variation between the minimum and maximum TMP values) along the membrane for larger flux, $Q$, and lower permeabilities, $k$. Therefore under constant flux in direct-flow filtration, this suggests that using low-permeability material and operating at low fluxes results in the filtration area being more evenly used. 
(a)

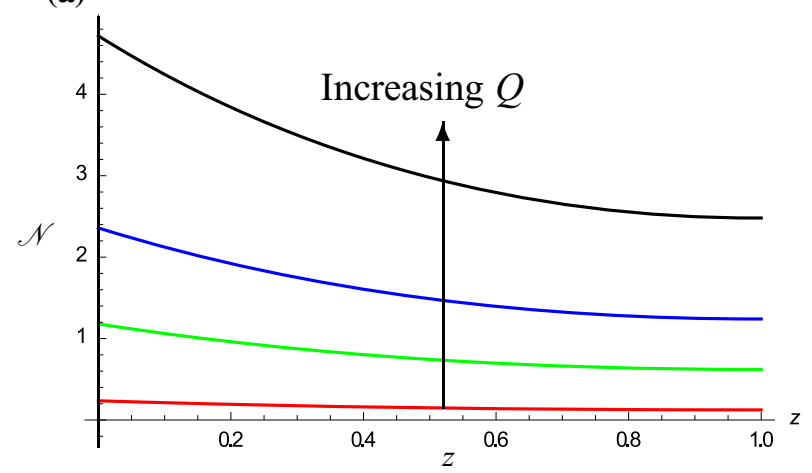

(b)

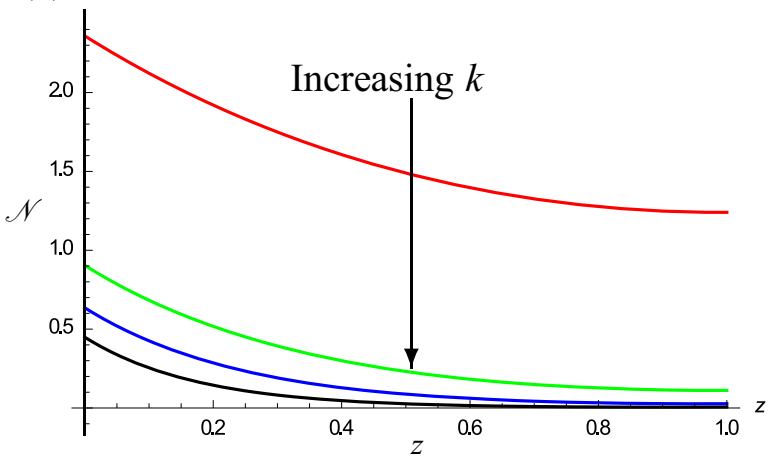

(c)

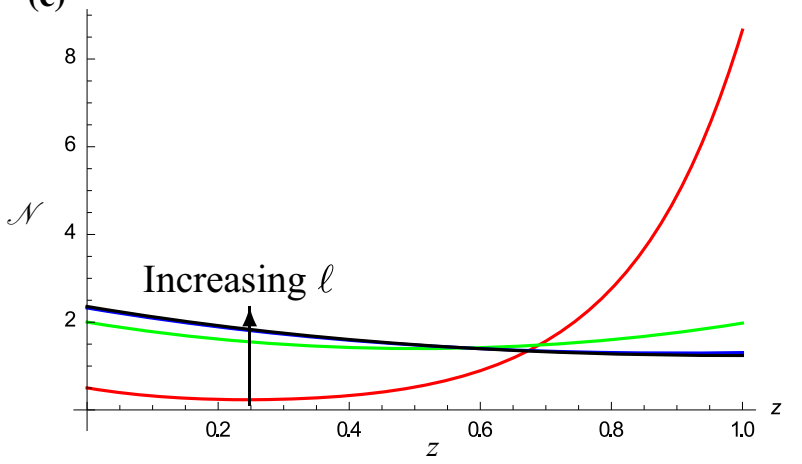

Fig. 6 Profiles of transmembrane pressure difference (TMP), $\mathcal{N}(z)=\left.(p-q)\right|_{r=1}$, in a capped axisymmetric pipe. Variations with a flux, $Q$ (with $\ell=1, k=0.1$ ); b permeability, $k$ (with $Q=1, \ell=1$ ); and $\mathbf{c}$ module spacing parameter, $\ell$ (with $Q=1, k=0.1$ ). In each figure, the varying quantity assumes the values $0.1,0.5,1,2$, plotted in red, green, blue and black, respectively. (Color figure online)

The TMP plots in the axisymmetric pipe (Fig. 6c) show qualitative similarities to that of the 2D channel (Fig. 5c) but differ in detail, which lead us to focus on the geometrical differences. For 2D channels, the module spacing parameter $\ell=D / H$ could also be counted as a cross-sectional area ratio of Region 2 to Region 1 . However, the situation differs in a pipe configuration: $\ell=D / R=1$ does not indicate an equal cross-sectional area for the inside and outside of the fibre. Therefore, we define another dimensionless module spacing parameter, $\gamma$, for the pipe configuration on an area basis

$\gamma=\frac{\text { Area outside the fibre }}{\text { Area inside the fibre }}=2 \ell+\ell^{2}$.

We now focus on the geometrical configuration of this device, characterized through the areal spacing parameters, $\ell$ (in 2D) and $\gamma$ (in 3D). For small areal spacing parameters, the TMP is low for most of the membrane channels and then rapidly rises to a high value as the capped end is approached $(z=1)$. In this case, the majority of filtration occurs at the end of the fibre and highlights the constraints that the impermeable capped end enforces on the TMP. In Fig. 7, the accumulated filtration volume percentage, $V_{c}$, clearly demonstrates the restrictions that arise when the module spacing parameter is too small. With a spacing parameter of 0.1 , there is almost no flux through the membrane wall over the first $95 \%$ of the length and all of the filtration essentially occurs in the last $5 \%$ near the impermeable capped end. For both $2 \mathrm{D}$ and $3 \mathrm{D}$, this is a situation to be avoided since a large area of the membrane is not being utilized and the very high local permeate flux at the end would lead to intense particle deposition. It is important to note that when the module spacing parameter becomes too small the packing arrangement chosen (e.g. a hexagonal or square array) in 3D plays a role. At this point our symmetry boundary conditions will no longer be appropriate and we would need to solve for the flow in the complete array using computational fluid dynamics 
(a) $V_{c}$

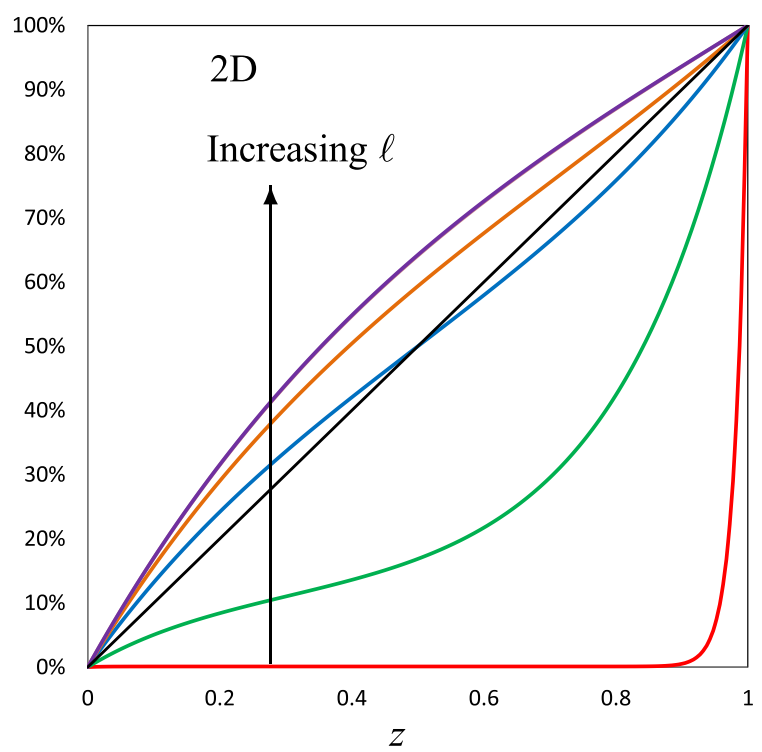

(b) $V_{c}$

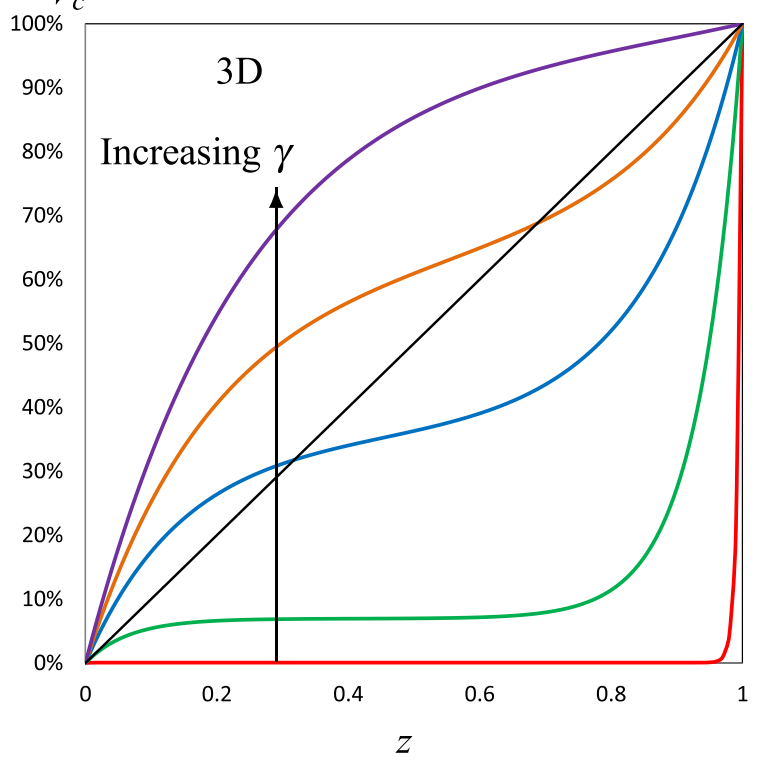

Fig. 7 The cumulative fluid volume transported through the fibre walls, $V_{c}(\%)$, versus axial distance, $z$, in a a $2 \mathrm{D}$ channel and $\mathbf{b}$ an axisymmetric pipe for various values of module spacing parameters ( $\ell$ and $\gamma$ respectively). In both cases, the varying quantity assumes the values $0.1,0.5,1,1.5$ and 5 , respectively at $Q=1$ and $k=1$. (Optimal performance corresponds to a linearly increasing cumulative flux, which is also shown as an additional solid straight line for comparison.)

tools. When the module spacing parameter is large, the feed channels or fibres are widely separated, and any further increase in the separation will have only a small effect on the flux profiles.

In the opposing regime of large areal spacing parameter, one should also note that in both 2D and 3D plots (Fig. 7), the profiles of percentage volume of cumulative filtered fluid will overlap when the module spacing parameter is large (i.e. $\ell=5$ with $\ell=10$ and $\gamma=5$ with $\gamma=10$ ). When $\ell$ in 2D or $\gamma$ in 3D are large (typically greater than 2), the feed channels or fibres are widely separated and thus further increases of $\ell$ or $\gamma$ larger than 2 will have a very small effect on the flux profile. Continuing to increase the module spacing parameter value beyond approximately 3 has only a small influence on the TMP plots and the overall filtration process (see blue and black lines in Figs. 5c and 6c).

The results of our analysis show that when fibres are packed too closely to one another the membrane area is not effectively used in a uniform manner. However, when the fibres are spaced far apart, although the area is more uniformly used, fewer fibres can be packed into a single direct-flow device. We therefore turn our attention to the question of how closely the fibres should be placed to optimize the operating efficiency of a direct-flow device. We frame this mathematically by introducing the design variable

$\alpha=\frac{P_{0}}{P_{m}} \frac{Q}{\left(1+S_{p}\right)}$,

where $Q$ is the fluid flux as defined earlier, $P_{m}$ is the maximum TMP for a chosen set of conditions, $S_{p}$ is the dimensionless module spacing parameter ( $\ell$ in $2 \mathrm{D}$ and $\gamma$ in 3D), and $P_{0}$ is the maximum operating pressure. The term $Q / P_{m}$ is a constant for a given fibre, and equates to a mean dimensionless permeability. Thus, $P_{0} Q / P_{m}$ expresses the productive capacity for a given set of conditions. Overall, the parameter $\alpha$ is a measure of the productive capacity per unit size of the module, i.e. a module productivity factor. We now wish to determine the operating regime that maximizes this factor.

We find that an optimal module spacing parameter $S_{p}$ exists that maximizes the module productivity in both the 2D and 3D filtration systems (in particular we find $S_{p}^{\mathrm{opt}} \approx 1$ in $2 \mathrm{D}$ and $S_{p}^{\mathrm{opt}} \approx 1.24$ in 3D, see Fig. 8). 
(a)

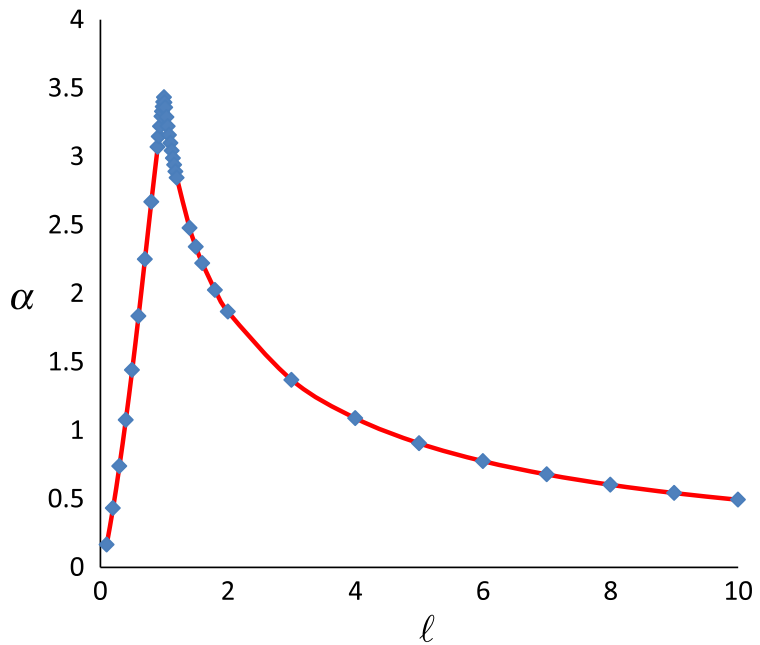

(b)

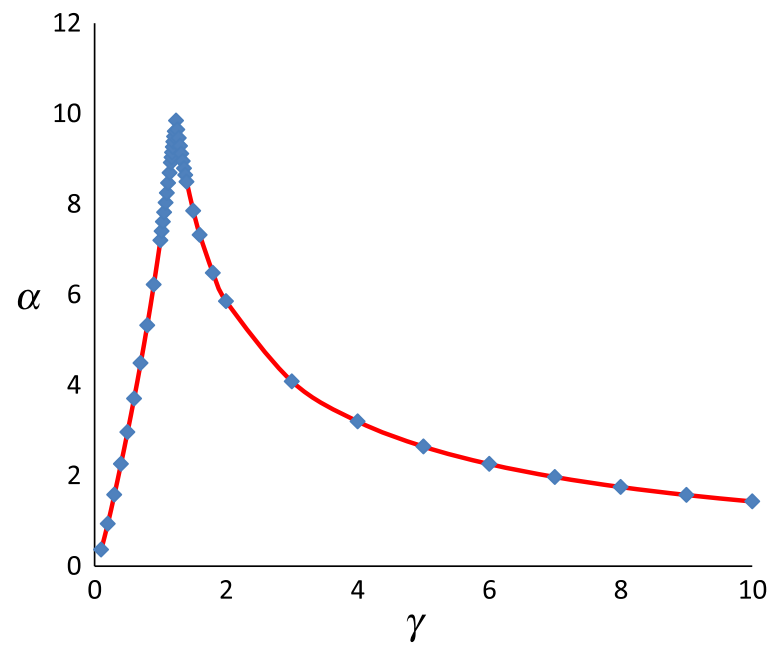

Fig. 8 The normalized module productivity, $\alpha$, versus different values of dimensionless module spacing parameters a $\ell$ in a $2 \mathrm{D}$ channel and $\mathbf{b} \gamma$ in an axisymmetric pipe respectively). In both cases, $Q=1, k=1$

We also quantify the uniformity of the filtration behaviour through the parameter $\varphi$ :

$\varphi=1-\frac{2}{V_{\text {final }}} \int_{0}^{1} \Delta V \mathrm{~d} z$

where $\Delta V(z)=\left|V_{\text {ideal }}-V_{c}(z)\right|$ with $V_{\text {final }}$ the total filtration volume, $V_{\text {ideal }}=z V_{\text {final }}$ the cumulative volume that would be filtered along the membrane under strictly uniform (idealized) filtration, and $V_{c}$ is the actual cumulative volume that has been filtered (see Fig. 7). When $\varphi=1$ the system filters the fluid uniformly across the entire fibre medium; when $\varphi=0$ all the fluid is filtered only at a single position in the fibre.

At low module spacing parameters, the uniformity of the filtration behaviour is low and increases as $S_{p}$ increases (Fig. 9). As for the module productivity, $\alpha$, we find that the filtration uniformity, $\varphi$, is optimized for module spacing parameter values of around 1 both in the $2 \mathrm{D}$ channel and axisymmetric pipe systems. In a $2 \mathrm{D}$ channel, we find that $\varphi>0.8$ for all $\ell \gtrsim 0.7$ and the peak occurs at $\ell \approx 1$ where $\varphi \approx 0.95$. This is consistent with Fig. 7a, which indicates the most uniform filtration performance when $\ell$ is near 1 . For a pipe, $\varphi>0.7$ for $0.95 \lesssim \gamma \lesssim 1.9$ and the peak occurs at $\gamma \approx 1.3$ where $\varphi \approx 0.82$. The values of the spacing parameter that create $95 \%$ uniformity in a 2D channel and $82 \%$ uniformity in pipe are also close to those that maximize the module productivity factor, $\alpha$. If one continuously increases $S_{p}$ beyond unity, the uniformity of the filtration behaviour will begin to fall again, asymptoting to the value attained for an individual fibre surrounded by an infinite bath of fluid.

The uniformity in filtration is also influenced by the membrane permeability, $k$, with smaller permeabilities giving rise to more uniform filtration (Fig. 10). However, one should avoid too low a permeability in a real engineering situation as this will result in either low throughputs or the need for a high inlet pressure. When the wall permeability is large, the central portion of the filter will contribute little to the overall filtration, with the membrane surface near the beginning and the end providing the main contribution, as seen in Fig. 10a. For example, when $k=10$, around $30 \%$ of the total flux passes through the membrane in the portion $0 \leq z \leq 0.2$; filtration then decreases with a small TMP, before the majority of the remaining fluid is filtered in the region $z \gtrsim 0.8$.

The pump-driving pressure is directly proportional to the flux, $Q$, and decreases with increasing permeability. As the module spacing parameter decreases, more of the fluid passes through a smaller area of the membrane, which also results in a higher operating pressure. Therefore, optimal direct-flow modules with a capped end should operate at low fluxes, be manufactured from a material with a relatively low permeability, and be arranged such that 

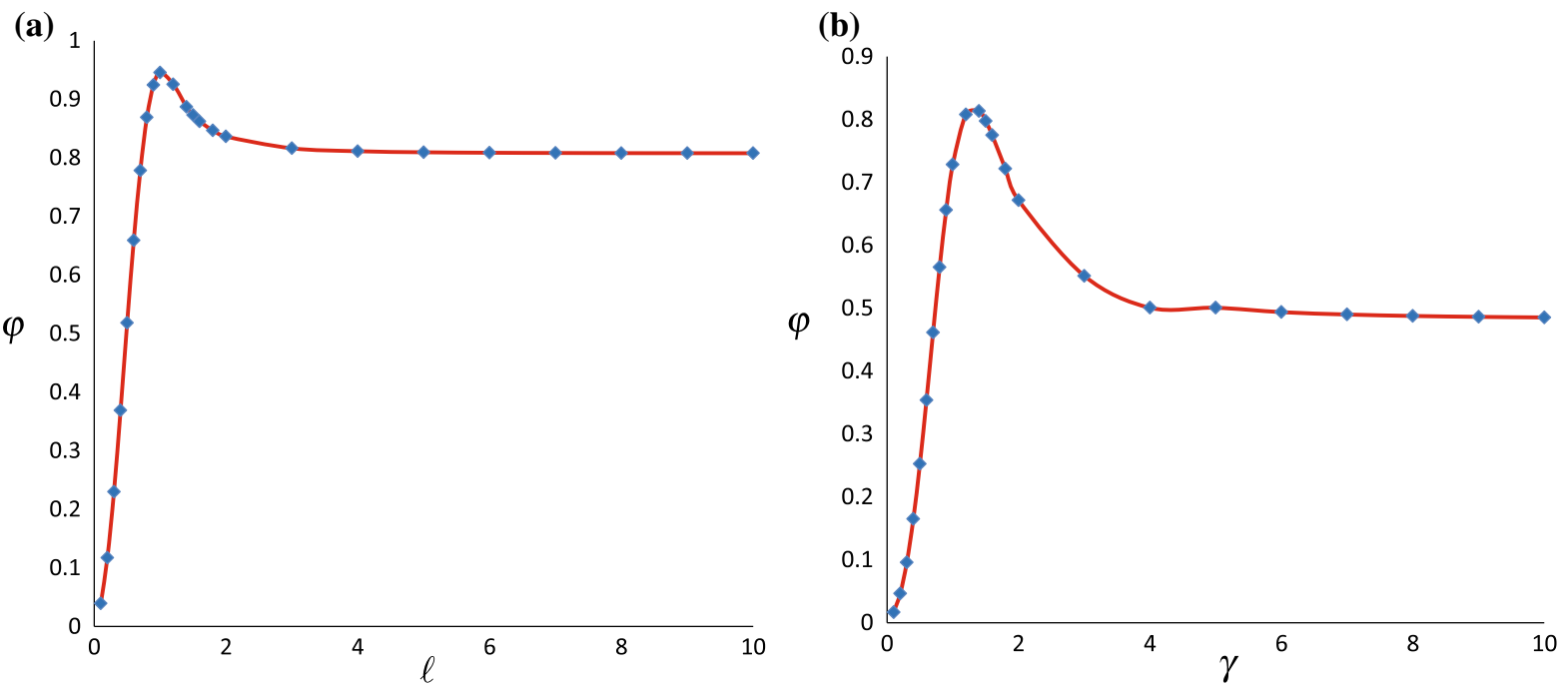

Fig. 9 The measure of the uniformity of the overall filtration, $\varphi$, for $\mathbf{a}$ a $2 \mathrm{D}$ channel versus $\ell$ and $\mathbf{b}$ an axisymmetric pipe versus $\gamma$, for a filtration setup in which $Q=1$ and $k=1$
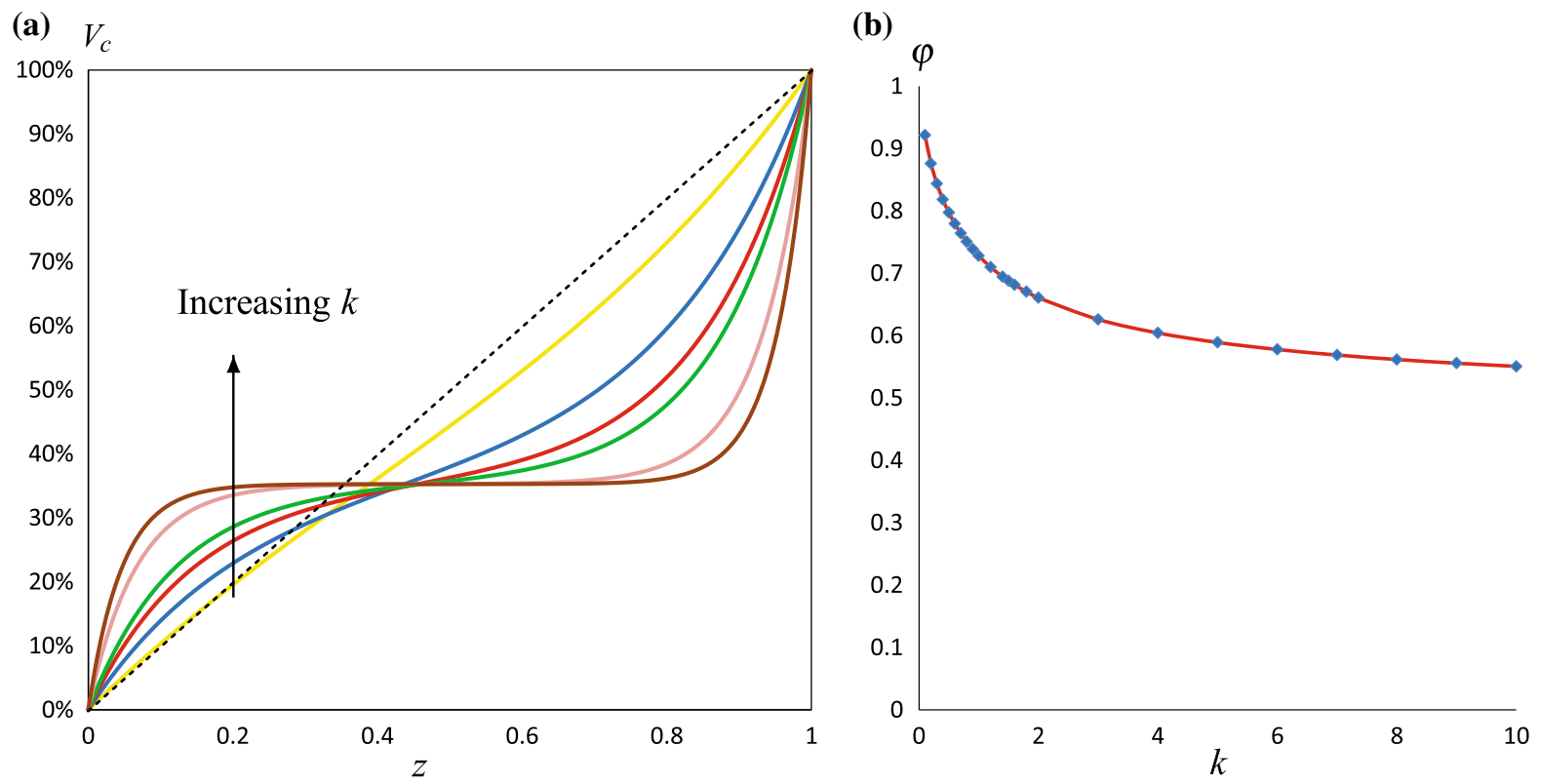

Fig. 10 a The cumulative fluid volume transported through the fibre walls (\%) versus axial distance $(z)$ in an axisymmetric pipe with different values of permeability $k$; $\mathbf{b}$ The measure of the uniformity of the overall filtration, $\varphi$, in a pipe versus permeability $k$. In $\mathbf{a}$, the varying quantity assumes the values $0.1,0.5,1,1.5,5$, and 10 , respectively (the black dashed line depicts the optimal filtration case). In both cases, $Q=1$ and $\gamma=1$

the ratio of the inside fibre to the outside is approximately unity. Such modules would ensure a relatively uniform filtration across the entire available fibre area in the system.

\section{Conclusions}

We have derived a mathematical model for the fluid flow in a direct-flow filtration module composed of a series of hollow fibres arranged within a single device, each with a capped end. The main focus of this work was to inves- 
tigate the influence of the proximity of neighbouring fibres on the flow behaviour and the filtration efficiency. This generalizes the models developed for a single hollow fibre with a blocked end [14] and models where neighbouring fibres are adjacent to one another [15].

The model is able to predict the operating regimes that optimize the uniformity of fluid transport across the whole membrane area, thus maximizing the efficiency of operation. We defined a module spacing parameter based on the ratio of cross-sectional areas of the feed and permeate side. We showed that, to maximize the uniformity of use of the filtration area, dead-end filter modules should operate at low fluxes, be composed of a filter material with a relatively low permeability and have neighbouring modules spaced such that the ratio of volumes on the feed and permeate side are approximately equal (i.e. module spacing parameter values of approximately unity).

For small module spacing parameters, we showed that a large portion of the available membrane space is not utilized, and the majority of the filtration occurs at or near the impermeable capped end. Module spacing parameters of around 1 will result in a close to optimal distribution of available membrane area in a 2D channel and 3D pipe module, when operating in a regime that optimizes the pressure applied and the space occupied by the system. The lower the permeability the higher the uniformity in the filtration system. However, this needs to be weighed against the fact that as the permeability falls a higher pump pressure is required to drive the fluid across the filter.

We have considered an important metric and proposed a strategy for optimizing the process for this metric. In principle, other factors may play a role, principally cost. Other functions could be constructed with respect to other optima which may be determined in a similar manner. The results of this paper should be useful in the future design of direct-flow devices and offers a deeper understanding of the filtration process in such a device. However, one should also be aware that what is uniform or reasonably uniform for this system may not be uniform for when the flow is reversed (backflow) in the same system. For evaluation of the overall filtration cycle (direct filtration followed by short sharp backflow), further research must be carried out to investigate the backflow in the same modules [27].

Acknowledgments J.G.H. thanks the Bracken Bequest for support during the work for this publication. Q.X. acknowledges the financial support from the China Scholarship Council. I.M.G. gratefully acknowledges the support from the Royal Society through a University Research Fellowship.

Open Access This article is distributed under the terms of the Creative Commons Attribution 4.0 International License (http:// creativecommons.org/licenses/by/4.0/), which permits unrestricted use, distribution, and reproduction in any medium, provided you give appropriate credit to the original author(s) and the source, provide a link to the Creative Commons license, and indicate if changes were made.

\section{References}

1. Bowen WR, Jenner F (1995) Theoretical descriptions of membrane filtration of colloids and fine particles: an assessment and review. Adv Colloid Interface Sci 56:141

2. Davis RH (1992) Modelling of fouling of crossflow microfiltration membranes. Sep Purif Methods 21:75

3. Noble RD, Stern SA (1995) Membrane separations technology: principles and applications, vol 2. Elsevier, Amsterdam

4. Vasan S, Field RW, Cui Z (2006) A Maxwell-Stefan-Gouy-Debye model of the concentration profile of a charged solute in the polarisation layer. Desalination 192(1):356

5. Van de Ven W, Vant Sant K, Pünt I, Zwijnenburg A, Kemperman A, Van der Meer W, Wessling M (2008) Hollow fiber dead-end ultrafiltration: influence of ionic environment on filtration of alginates. J Membr Sci 308(1):218

6. Bessiere Y, Abidine N, Bacchin P (2005) Low fouling conditions in dead-end filtration: evidence for a critical filtered volume and interpretation using critical osmotic pressure. J Membr Sci 264(1):37

7. Blankert B, Betlem BH, Roffel B (2006) Dynamic optimization of a dead-end filtration trajectory: blocking filtration laws. J Membr Sci 285(1):90

8. Grenier A, Meireles M, Aimar P, Carvin P (2008) Analysing flux decline in dead-end filtration. Chem Eng Res Des 86(11):1281

9. Balannec B, Vourch M, Rabiller-Baudry M, Chaufer B (2005) Comparative study of different nanofiltration and reverse osmosis membranes for dairy effluent treatment by dead-end filtration. Sep Purif Technol 42(2):195

10. Li NN, Fane AG, Ho WW, Matsuura T (2011) Advanced membrane technology and applications. Wiley, New York

11. Field RW, Pearce GK (2011) Critical, sustainable and threshold fluxes for membrane filtration with water industry applications. Adv Colloid Interface Sci 164(1):38

12. Pearce GK (2011) UF/MF membrane water treatment: principles and design. Water Treatment Academy, Bangkok

13. Karode S (2001) Laminar flow in channels with porous walls, revisited. J Membr Sci 191(1-2):237 
14. Hurwitz MF (1989) Flows through porous tubes-the end cap problem. In: Proceedings from mathematical problems in industry workshop

15. Sanaei P, Richardson GW, Witelski TP, Cummings LJ (2016) Flow and fouling in a pleated membrane filter. J Fluid Mech 795:36

16. Griffiths IM, Howell PD, Shipley RJ (2013) Control and optimization of solute transport in a thin porous tube. Physics Fluids 25(3):033101 (1994-present)

17. Bacchin P, Aimar P, Field RW (2006) Critical and sustainable fluxes: theory, experiments and applications. J Membr Sci 281(1):42

18. Guerra A, Jonsson G, Rasmussen A, Nielsen EW, Edelsten D (1997) Low cross-flow velocity microfiltration of skim milk for removal of bacterial spores. Int Dairy J 7(12):849

19. Beolchini F, Cimini S, Mosca L, Veglio F, Barba D (2005) Microfiltration of bovine and ovine milk for the reduction of microbial content: effect of some operating conditions on permeate flux and microbial reduction. Sep Sci Technol 40(4):757

20. Herterich JG, Griffiths IM, Vella D, Field RW (2014) The effect of a concentration-dependent viscosity on particle transport in a channel flow with porous walls. AIChE J 60(5):1891

21. Van der Bruggen B, Vandecasteele C, Van Gestel T, Doyen W, Leysen R (2003) A review of pressure-driven membrane processes in wastewater treatment and drinking water production. Environ Prog 22(1):46

22. Kedem O, Katchalsky A (1958) Thermodynamic analysis of the permeability of biological membranes to non-electrolytes. Biochim Biophys Acta 27:229

23. Beavers GS, Joseph DD (1967) Boundary conditions at a naturally permeable wall. J Fluid Mech 30:197

24. Altena FW, Belfort G (1984) Lateral migration of spherical particles in porous flow channels: application to membrane filtration. Chem Eng Sci 39(2):343

25. Shipley RJ, Waters SL, Ellis MJ (2010) Boundary conditions at a naturally permeable wall. Biotechnol Bioenergy 107:382

26. Probstein RF (1989) Physicochemical hydrodynamics. an introduction. Butterworths, Boston

27. Stana R (1974) Reverse pressure cleaning of supported semipermeable membranes. US Patent $3,853,756$ 